\title{
CHARMONIA AT FINITE MOMENTA IN A DECONFINED PLASMA *
}

\author{
S. DATTA, F. KARSCH AND S. WISSEL \\ Fakultät für Physik, Universität Bielefeld, D-33615 Bielefeld, Germany. \\ E-mail: saumen,karsch,wissel@physik.uni-bielefeld.de \\ P. PETRECZKY ${ }^{\dagger}$ \\ Brookhaven National Laboratory, Upton, NY 11973, USA. \\ E-mail: petreczk@quark.phy.bnl.gov \\ I. WETZORKE \\ NIC/DESY Zeuthen, Platanenallee 6, D-15738 Zeuthen, Germany. \\ E-mail: Ines.Wetzorke@desy.de
}

\begin{abstract}
Lattice studies of charmonium systems have indicated that in a deconfined gluonic plasma ground state charmonia survive as bound states upto temperatures $\sim 2 T_{c}$. After surveying the methodologies used in reaching these results, we examine the behavior of these systems when the bound state is in motion with respect to the heatbath frame. We find that the finite momenta charmonia show medium modifications when the medium is deconfined; in particular, a modification of the energy-momentum dispersion relation is indicated.
\end{abstract}

The study of strongly interacting matter at high temperatures is important both for understanding QCD and for cosmological applications, and is a very active field with experimental data available from relativistic heavy ion collisions. Charmonium systems have been of great interest to the relativistic heavy ion community ever since Matsui and Satz, based on potential model studies, predicted that charmonium bound states cannot survive in a deconfined plasma, and suggested $J / \psi$ as a probe of the confinement status of the plasma ${ }^{1}$. Later, more detailed potential model studies suggested that

\footnotetext{
${ }^{*}$ This research is supported by BMBF under grant no. 06BI102, and by DOE under contract DE-AC02-98CH10886.

${ }^{\dagger}$ Goldhaber and RIKEN fellow
} 
all charmonium bound states dissolve before or pretty soon after deconfinement ${ }^{2}$. However, a direct lattice investigation of the hadronic correlators have revealed that the ground state charmonia $\left(\eta_{c}\right.$ and $\left.J / \psi\right)$ survive the deconfinement transition in pure gauge theory ${ }^{3,4,5}$, and may dissolve only at temperatures $\sim 2 T_{c}$.

Both for understanding the mechanism of bound state dissolution in the plasma and for study of charmonium abundance in experiments, it is important to also know the fate of these bound states when they not at rest but moving with respect to the heatbath frame. The lack of covariance in finite temperature system means the pole of the inverse propagator,

$$
\Delta^{-1}\left(p_{0}, p, T\right)=p_{0}^{2}+p^{2}+m^{2}+\operatorname{Re} \Pi(p, T)+i \operatorname{Im} \Pi(p, T)
$$

can be a generic function of spatial momentum $\mathrm{p}$, leading possibly to a modification of the energy-momentum dispersion relation and a momentum dependent decay width. Such modifications have been discussed in different models for pions in hadron gas ${ }^{6}$, and for decay width of $J / \psi$ in hadron gas ${ }^{7}$. Here, we present a preliminary report of a direct lattice investigation into the fate of the ground state charmonia with a finite spatial momentum (in the heatbath frame). In this report, we will restrict ourselves to comparatively moderate temperatures $\leq 1.5 T_{c}$.

Direct lattice investigations of the fate of the charmonium states involve analyzing the Matsubara correlators,

$$
G_{H}(\tau, \vec{p}, T)=\left\langle J_{H}(\tau, \vec{p}) J_{H}^{\dagger}(0,-\vec{p})\right\rangle_{T}
$$

where $J_{H}$ is the suitable mesonic operator, projected on the state with spatial momentum $\vec{p}$. For studying the $\eta_{c}$ and the $J / \psi$, we use the pointpoint operators $\bar{c} \gamma_{5} c$ and $\bar{c} \gamma_{\mu} c$, respectively. For the vector channel, here we consider the transverse component only. Through analytical continuation, the Matsubara correlator can be related to the hadronic spectral function by an integral equation:

$$
G(\tau, \vec{p}, T)=\int_{0}^{\infty} d \omega \sigma(\omega, \vec{p}, T) \frac{\cosh (\omega(\tau-1 / 2 T))}{\sinh (\omega / 2 T)} .
$$

While the inversion of Eq. (3) to get the spectral function from the correlators measured at a finite set of points is clearly an ill-defined problem, some progress has been made in the last few years towards tackling such problems using Bayesian tools. In the maximum entropy method ${ }^{8}$ the positivity of the spectral function and information about the asymptotic behavior is used to find the most probable spectral function given data. Analysis of the hadronic correlators at low temperatures (below $T_{c}$ ) 
reveal a complicated structure in the high energy regime of the spectral functions, dominated by lattice artifacts ${ }^{3}$. We use this structure as part of the asymptotic information in our analysis at higher temperatures ${ }^{3}$.

Useful information about possible change of state with deconfinement can be obtained by comparing the correlators measured above $T_{c}$ with the correlators reconstructed from the spectral function below $T_{c}$. We define $G_{\text {recon, } T^{*}}(\tau, \vec{p}, T)$ by substituing $\sigma(\omega, \vec{p}, T)$ in Eq. (3) with $\sigma\left(\omega, \vec{p}, T^{*}\right.$ where $T^{*}$ is usually taken as the smallest temperature below $T_{c}$ available to us. This takes into account the trivial temperature dependence of the kernel and therefore, any deviation of $G(\tau, \vec{p}, T)$ from $G_{\text {recon, } T^{*}}(\tau, \vec{p}, T)$ will indicate a temperature modification of the spectral function ${ }^{3}$. In Fig. 1 the results of such a comparison are shown for zero momentum correlators. For the pseudoscalar channel, Fig. 1 shows that the reconstructed correlator explains the measured correlators completely upto temperatures of $1.5 T_{c}$, indicating that there is no significant change for these states upto this temperature. The spectral function extracted from the correlators, shown in the right, confirm this. For $J / \psi$, some small deviation of the measured correlators is seen from the reconstructed correlators, indicating a possible widening ${ }^{4}$ or mass shift. Here we added the three (spatial) components of the vector, and the behavior is somewhat different from the four component correlator in Ref. [3] due to the change in contribution from electric conductivity below and above $T_{c}$.
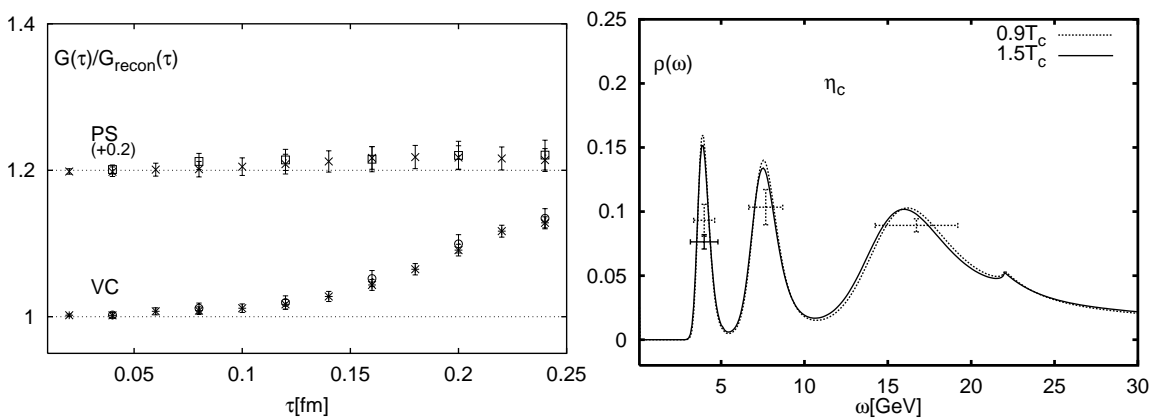

Figure 1. Comparison of zero momentum correlators at $1.5 T_{c}$ for vector and pseudoscalar charmonia with those reconstructed from spectral functions at $0.75 T_{c}$ (left). The stars and crosses correspond to a lattice with lattice spacing of $0.02 \mathrm{fm}$ and the the open symbols correspond to a lattice with lattice spacing $0.02 \mathrm{fm}$. (Right) Reconstructed spectral functions at $1.5 T_{c}$ and $0.9 T_{c}$ for the pseudoscalar channel. Plotted is the rescaled spectral function $\rho(\omega)=\sigma(\omega) / \omega^{2}$. The default model included the peak structure at high $\omega$, which is dominated by lattice artifacts. 
For investigation of the finite momenta charmonia, we use the same analysis methods as those at zero momentum described above. Comparison of the finite momentum correlators $G(\tau, \vec{p}, T)$ at 1.1 and $1.5 T_{c}$ with the reconstructed correlators from $\sigma(\omega, \vec{p}, T)$ are presented in Fig. 2. It can be seen that with increasing spatial momentum, the correlators above $T_{c}$ show larger deviations from the reconstructed correlators, signalling a medium effect already at $1.1 T_{c}$ and increasing with temperature.

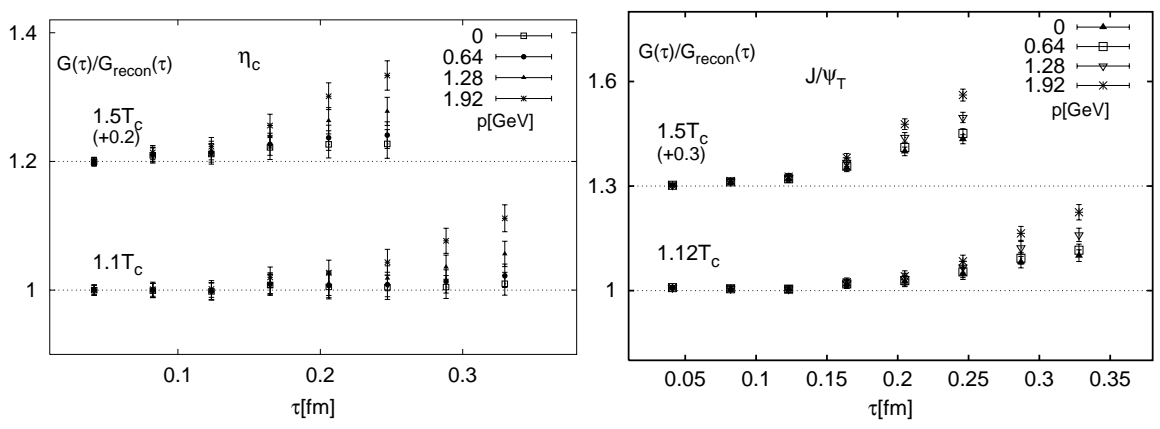

Figure 2. Comparison of correlators at $1.1 T_{c}$ and $1.5 T_{c}$ for pseudoscalar and (transverse) vector charmonia with those reconstructed from spectral function at $0.75 T_{c}$.

In order to get further insight into the nature of the medium effect, we used maximum entropy analysis for a direct reconstruction of $\sigma(\omega, \vec{p}, T)$ above $T_{c}$. As described before, for the reconstruction we use the high energy structure of the spectral function obtained at $0.75 T_{c}$ as part of the prior information. The spectral functions at $0.75 T_{c}$ and $1.1 T_{c}$, using such a default model, are shown in Fig. 3. Here, in order to avoid any systematics due to the small spatial extent and limited number of data points at the higher temperature, we use the same physical extent and same number of data points both below and above $T_{c}$, by omitting points at the center for the lower temperature.

Figure 3 shows two interesting features. First, a strong signal from the ground state shows up in the spectral function at $1.1 T_{c}$ even for momenta $\sim 2 \mathrm{GeV}$. Little change in peak strength is seen on crossing deconfinement, for any of the momenta. Second, there is an indication of a slower movement of the peak position above $T_{c}$, indicating a modification of the energymomentum dispersion relation. These trends continue at $1.5 T_{c}$, with a significant bound state showing up with a modified dispersion relation.

The $J / \psi$ moving in the heatbath frame sees more energetic gluons than 

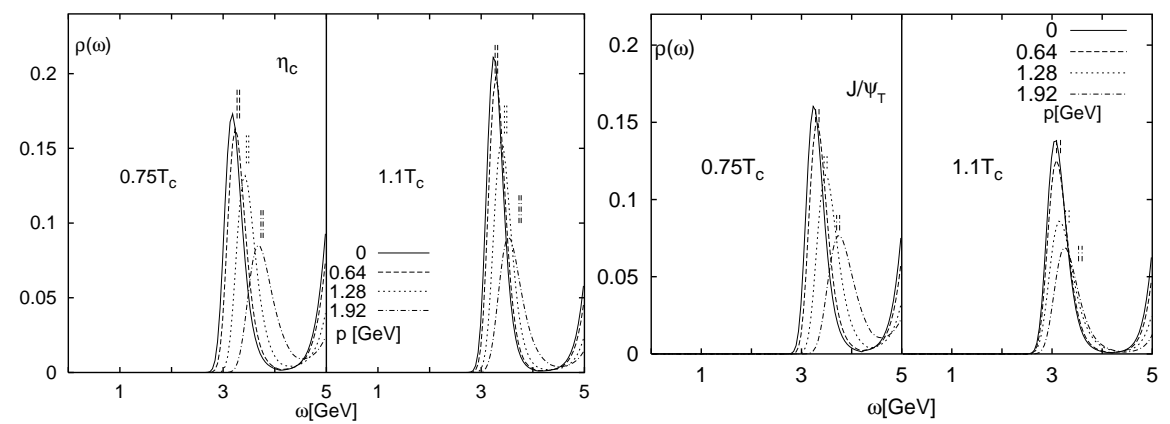

Figure 3. Spectral functions at $0.75 T_{c}$ and $1.1 T_{c}$ for different spatial momenta, for lattices with lattice spacings of $0.04 \mathrm{fm}$. The small horizontal bars indicate the expected shift of the peak using relativistic dispersion relation. The same temporal extent has been used at both the temperatures.

the $J / \psi$ at rest. The fact that $J / \psi$ moving at such momenta show a statistically significant peak indicates a stongly nonperturbative nature of the plasma. We would like to clarify that we are investigating here $J / \psi$ states in an equilibriated plasma. This is very different from earlier phenomenological studies concerned with survival of $J / \psi$ with large trasnverse momentum from a finite sized plasma, where a large transverse momentum may lead to the $J / \psi$ spending a smaller time in the plasma, resulting in a larger survival probability ${ }^{9,2}$. However, such studies also need to take into account the fact that the in-medium dispersion relation for the $J / \psi$ will be modified. A modified dispersion relation may also explain the large temperature dependence observed in the screening mass of the $J / \psi$ and the $\eta_{c}{ }^{3}$; however, a quantitative statement will require a much more exact determination of the dispersion relation than is available at present.

\section{References}

1. T. Matsui and H. Satz, Phys. Lett. B 178, 416 (1986).

2. F. Karsch and H. Satz, Z. Phys. C 51, 209 (1990).

S. Digal et al., Phys. Lett. B514, 57 (2001).

3. S. Datta et al., Phys.Rev. D 69, 094507 (2004).

4. T. Umeda et al., hep-lat/0211003.

5. M. Asakawa and T. Hatsuda, Phys. Rev. Lett. 92, 012001 (2004).

6. E. Shuryak, Phys.Rev. D 42, 1764 (1990).

7. K. Haglin and C. Gale, Phys. Rev. D 63, 065201 (2001).

8. M. Jarrel and J. E. Gubernatis, Phys. Rept. 269, 133 (1996).

M. Asakawa et al., Prog. Part. Nucl. Phys. 46, 459 (2001).

9. M.-C. Chu and T. Matsui, Phys. Rev. D 37, 1851 (1988). 